\title{
Modellus: Interactive computational modelling to improve teaching of physics in the geosciences
}

\author{
Rui G. M. Neves ${ }^{\text {a }}$, Maria C. Neves ${ }^{b}$, Vítor Duarte Teodoro ${ }^{c}$ \\ ${ }^{a}$ Unidade de Investigação Educação e Desenvolvimento (UIED) e Departamento de \\ Ciências Sociais Aplicadas (DCSA), Faculdade de Ciências e Tecnologia, Universidade \\ Nova de Lisboa (FCT/UNL), Monte da Caparica, 2829-516 Caparica, Portugal. \\ Email: rgn@fct.unl.pt. \\ ${ }^{\mathrm{b}}$ IDL, FCT, Universidade do Algarve (UAlg), Portugal. \\ Email: mcneves@ualg.pt. \\ ${ }^{c}$ UIED-DCSA, Faculdade de Ciências e Tecnologia, Universidade Nova de Lisboa \\ (FCT/UNL), Portugal. \\ Email: vdt@fct.unl.pt.
}

\begin{abstract}
Many aspects of modern research and other professional activities in the geosciences require advanced knowledge about mathematical physics models and scientific computation methods and tools. In-depth meaningful learning of such knowledge skills is a difficult cognitive process which involves developing strong background knowledge of physics, mathematics and scientific computation appropriately contextualized in the geosciences themes. In this paper we describe an interactive engagement teaching approach that is based on Modellus, a freely available computer software system allowing (1) mathematical modelling ranging from explorative to expressive modelling, (2) the introduction of scientific computation without requiring the development of a working knowledge of programming and (3) the simultaneous manipulation and analysis of several different model representations, namely, tables, graphs and animations with interactive objects having properties defined in a visible and modifiable mathematical model. As examples of application, with insights for the development of other activities in a wide range of geosciences courses, we discuss a set of interactive computational modelling activities for introductory meteorology we have implemented in undergraduate university courses.
\end{abstract}

Keywords: Modelling; computational geosciences; interactive engagement; meaningful learning; physics; meteorology

\section{Introduction}

Physics and mathematics are fundamental subjects for the development of knowledge in geosciences and related industrial or technological fields. Their modern epistemologies, like those of geosciences, involve interactive modelling processes that balance different elements from theory, scientific computation and experimentation. However, the majority of current introductory physics and mathematics courses for the diverse areas of science do not always reflect this range of epistemological characteristics. For example, introductory physics courses at university level, even when equipped with modern technologies, usually involve expositive theoretical lectures and recipe experimental laboratories and problem solving classes which brush through a very large number of topics. In general, these courses are considered too difficult and 
disappointing by many students and have low exam success rates. Also, for many students the acquired knowledge of physics and mathematics is fragmented and possesses numerous conceptual and reasoning weaknesses which persist after they pass their examinations (Halloun and Hestenes, 1985a, 1985b; McDermott, 1991). Furthermore, student expectations about physics often deteriorate after completing these courses (Redish et al., 1998). Similar learning problems with fundamental earth sciences concepts have also been documented (see, e.g., Libarkin and Anderson, 2005; Libarkin et al., 2005).

Many research efforts have been able to show in a wide range of contexts that learning processes can be effectively enhanced when students are embedded in atmospheres with interactive engagement activities that approximately recreate the cognitive involvement of scientists in modelling research activities (see, e.g., Blum et al., 2007; Handelsman et al., 2005; Klosko et al., 2000; Kortz et al., 2008; McConnell et al., 2006; McDermott and Redish, 1999; Meltzer and Thornton, 2012). Contrary to traditional instruction approaches which end up reducing the learning processes to a rote accumulation of facts or rules, these research-based approaches have shown to be able to engage students in interactive and exploratory learning processes that are better suited to promote knowledge performance and to resolve cognitive conflicts with prior knowledge associated to common sense beliefs and incorrect scientific ideas.

In many areas of the geosciences, professional modelling activities are often based on knowledge about advanced mathematical physics models which are rich in computational elements. In-depth acquisition of such knowledge involves cognitive processes which require the progressive development of a strong background in physics, mathematics and computer programming. At an introductory level, for example in the first two year cycle of university education, when such background is still forming, it is thus of interest to design research based geosciences curricula which integrate sets of relatively simple computational modelling activities involving computer software environments. In this paper we show how to use the computational modelling software Modellus to accomplish this goal. The examples we describe have been developed in the context of teaching activities we have implemented in introductory meteorology courses for first cycle undergraduate university students. These examples are illustrative and insightful of functionalities and potentialities which can be used to develop other computational modelling activities to teach mathematical physics models in a wide range of topics in geosciences courses. The objective is to give students the opportunity to improve their knowledge of physics, mathematics and scientific computation and simultaneously focus on the learning of the relevant geosciences concepts and processes.

\section{Teaching approach}

An effective application of Modellus in a geosciences classroom requires a carefully designed teaching strategy. In our field activities the courses are divided into three complementary components: lectures where the theoretical foundations were first introduced, paper and pencil problem-solving lessons, and the computational modelling classes based on Modellus. The goal is to create an interactive engagement environment based on approaches already shown to be highly effective to teach introductory physics in other contexts (see in particular, e.g., Beichner et al., 2007; Crouch et.al., 2007; Mazur, 1997; McDermott et al., 1996; McDermott and Shaffer, 2002; Neves et al., 2011).

To build this kind of environment students are organized in group teams of two or three. During each computational modelling class, the teams work on a set of activities, 
all of which are to be completed using only Modellus as computer modelling tool. These activities are designed to be interactive and exploratory learning experiences structured around specific topics and aim to set up an atmosphere for meaningful learning (see, e.g., Mintzes et al., 2005) where students approximately work as scientists do in modelling research activities. In class the student teams are motivated to analyse, discuss and solve the proposed activity problems on their own using the physical, mathematical and computational modelling guidelines provided by the class documentation and software resources. Note that these activities are appropriately articulated with the complementary theoretical and paper and pencil problem solving classes. In addition, the teams are not left working alone but continuously helped during the exploration of the activities to ensure adequate working rhythm with appropriate conceptual, analytical and computational understanding. Whenever necessary, global class discussions are conducted to keep the pace, to introduce new themes, to clarify any doubts on concepts, reasoning or calculations common to several teams and for students work presentations.

The supporting class documentation and software resources for the courses we have implemented included Modellus package examples and a set of activity PDF documents. For most of the class activities these PDF documents contained complete step-by-step instructions to build the Modellus mathematical models, animations, graphs and tables. However, some activities, including those for assessment, involved computational modelling problems with instructions having various challenging levels of incompleteness.

\section{Getting started with Modellus}

Modellus latest version is programmed in Java and is able to run in all operating systems. It is freely available and the installation instructions as well as the user manual can be found at the Modellus website ${ }^{1}$. The installation package includes many sample models and others are regularly added to the website which, in addition, contains several other supporting documents. In the rest of this section we briefly describe the most important elements of the graphical user interface and discuss the main steps to create a model with Modellus.

Upon starting the Modellus window interface (Fig. 1) displays at the top the Menu bar containing 9 ribbons labelled Home, Independent Variable, Model, Parameters, Initial Conditions, Graph, Table, Objects and Notes. The Mathematical Model, Graph, Table and Notes windows can be expanded, minimized or moved within the Workspace area. The Animation Control bar lies at the bottom and contains, for example, a green Play and Pause button, a red Reset button and blue buttons for Replay, One step back and One step forward. Fig. 1 also shows the Home definition panel which allows the user to change aspects of the global interface appearance, such as the interface language and the way numbers are displayed, or to introduce background grids or images in the Workspace. The model set up includes the following steps:

(1) Write the mathematical expressions in the Mathematical Model window. This window works just like a calculator and allows the user to program functions, differential equations and numerical iterations with standard mathematical notation. Modellus has a set of pre-defined functions whose syntax is displayed upon clicking on the Help button of the Model panel (Fig. 2).

\footnotetext{
${ }^{1}$ See the website page at http://modellus.fct.unl.pt.
} 
(2) Define the independent variable along with its interval domain and running numerical step in the Independent Variable ribbon. Note that it is not possible to define simultaneously more than one independent variable.

(3) Specify the parameters and initial conditions either directly in the Mathematical Model window or in the Parameters and Initial Conditions ribbons.

(4) Choose the representation options (Graph, Table or Animation). We emphasise that these different representations can be displayed simultaneously alongside the mathematical model, a fundamental advantage of Modellus to help building a full understanding of the underlying principles of a model.

- The Graph ribbon allows the user to define which variables are attributed to the graph horizontal and vertical axis, adjust the corresponding scales or select graph line properties like colour and thickness.

- The Table ribbon allows up to 8 column variable entries and the introduction of table data bars.

- The Animation representation is constructed with the set of animation objects available in the Objects ribbon. The object is first selected from the object's list (for example a Particle or Pen object) and then placed in the Animation area with a left mouse click. Whenever an animation object is created a new ribbon labelled Animate appears in the top Menu bar displaying the object definitions panel. The advantage of animation objects is that they are interactive. A simple example is to assign a mathematical model parameter to the Level Indicator and vary its value by sliding the Level Indicator bar.

(5) Perform the computation of the mathematical model over the independent variable domain. This is done by clicking on the green Play button at the bottom of the Modellus window (Fig. 1). The graph or animation is created as the simulation runs, while the progress indicator icon moves along the Animation Control bar. It can be paused at any time for observation and solution control. Changing the parameters at any stage during the simulation allows interactive exploration of the effects of such changes and encourages playing what-ifanalysis.

It is important to note that the interactive explorative activities can be accomplished in two different ways. The first is by defining simultaneous Cases (Fig. 2) which can be visualised at the same time. This kind of action can be done with any type of model depending on a set of parameters. The parameters phase space exploration is defined a priori using the case column options in the Parameters ribbon. The second kind of interactive explorative action involves changing parameters while the model is running. This action can only be done with iterative models generating numerical solutions that are functions of the independent variable because such numerical solutions are up-dated in real time as successive iterations are calculated. In the next section, while discussing geosciences applications, we illustrate some examples of interactive explorative activities.

\section{Examples of application}

To put in evidence the capabilities Modellus has for improved teaching of physics models in geosciences contexts we consider two examples: (1) the blackbody radiation 
laws, to illustrate the use of graphical representations and numerical integration, and (2) the gradient wind velocity law, to illustrate the use of animations.

\subsection{Blackbody radiation laws}

The laws for blackbody radiation are a standard introductory physics topic with numerous applications ranging from the study of energy transfer in the Earth's atmosphere to stellar astrophysics. In this computational modelling activity we propose to students an interactive exploration of Planck's law for the radiation power density function leading to Wien and Stefan-Boltzmann laws.

The starting step (Fig. 3) is to write in the Modellus Mathematical Model window the radiation power density function $B(\lambda)$ which is given by

$B(\lambda)=\frac{2 \pi h c^{2}}{\lambda^{5}\left(\mathrm{e}^{\frac{c h}{k \lambda T}}-1\right)}$,

where $\lambda$ is the radiation wavelength, $h$ is Planck's constant, $c$ is the speed of light, $k$ is the Boltzmann constant and $T$ is the temperature.

The parameters $h, c, k$ and $T$ are defined in the Parameters ribbon (Fig. 3). The radiation wavelength is chosen to be the independent variable and the next step is to define in the Independent Variable ribbon the adequate domain interval for $\lambda$. This provides an opportunity for students to verify the range of values relevant for atmospheric radiation, in particular, the visible (solar) and infrared (terrestrial) radiation intervals. In the same ribbon students can also define the numerical step $\Delta \lambda$ associated with $\lambda$. The following step is to represent $B(\lambda)$ in graphical form. In figure 3 we show a Graph window with 3 different curves corresponding to 3 different temperature cases, namely, $T=300 \mathrm{~K}, T=400 \mathrm{~K}$ and $T=500 \mathrm{~K}$. Figure 3 also shows the Table window where the values used to draw these curves can be explicitly displayed in table form.

One of the advantages of using numerical solutions is to give introductory level students the opportunity to deduce Wien and Stefan-Boltzmann laws without having to perform the corresponding analytic derivations which are beyond the learning scope of most introductory courses. To deduce Wien displacement law students start by selecting one of the graphs representing $B(\lambda)$ for a certain value of the temperature $T$, for example, $T=400 \mathrm{~K}$ (Fig. 4). Selecting Tangent Lines in the Graph ribbon and using the mouse to move it along the graph, it is possible to visualize the tangent at every point along the curve and read in the abscissa's axis the value of $\lambda$ for which this tangent is horizontal. At this point $\lambda_{\max }=7.26 \times 10^{-6} \mathrm{~m}$, the radiation power density attains its maximum value $B\left(\lambda_{\max }\right)=1.31 \times 10^{8} \mathrm{~W} / \mathrm{m}^{3}$. Students can then compute the product $\lambda_{\max } T$ and verify that the numerical result is approximately equal to the theoretical value of Wien's constant, $c \mathrm{~W}=2.898 \times 10^{-3} \mathrm{mK}$. Students can interactively check that the fit between the computed and the theoretical Wien constant is improved when a smaller numerical step $\Delta \lambda$ is used, and also that Wien's law is similarly obtained using another $B(\lambda)$ curve for a different value of $T$.

Finally, to deduce Stefan-Boltzmann law students use numerical integration to show that the power radiated per unit area $E$ satisfies

$E=\int_{0}^{+\infty} B(\lambda) \mathrm{d} \lambda=\sigma T^{4}$

where $\sigma=5.67 \times 10^{-8} \mathrm{~W} /\left(\mathrm{m}^{2} \mathrm{~K}^{4}\right)$ is the Stefan-Boltzmann constant. The integration is programmed in the Mathematical Model window using the instruction (Fig. 3) 
$E=\operatorname{last}(E)+B \times \Delta \lambda$

with the initial condition $E=0$. This is an application of the trapezoidal rule, a simple and useful numerical method for students just starting an introduction to scientific computation. The result of the integration can be visualized creating a Variable object ESB (Fig. 3) or plotting the graph of $E$ as a function of $\lambda$. In figure 3 we show this graph as it is created by a Pen in the Animation area. The curve represents the accumulated area below $B(\lambda)$ as $\lambda$ runs through its domain and students can verify that it approaches a constant value approximately equal to the product $\sigma T^{4}$.

\subsection{Gradient wind velocity}

The dynamics of atmospheric air is determined applying Newton's laws to each air particle. One of the simplest solutions of the equations of motion can be obtained when we consider steady state circular horizontal and frictionless atmospheric flow. In the northern hemisphere the balance between the pressure gradient force, the Coriolis force and the centrifugal force is given, respectively for low pressure and high pressure systems, by

$-f v+\frac{1}{\rho} \operatorname{gradp}-\frac{v^{2}}{R}=0$

and

$f v-\frac{1}{\rho} \operatorname{grad} p-\frac{v^{2}}{R}=0$

where $V$ is the speed of the air particle, $R$ is the curvature of the air particle trajectory, $\rho$ is the air density, gradp is the magnitude of the pressure gradient and $f$ is the Coriolis parameter defined as $f=2 \omega_{\mathrm{T}} \sin (\varphi)$ with $\omega_{\mathrm{T}}$ denoting the Earth's rotation angular velocity and $\varphi$ the latitude angle. At large scale synoptic low and high pressure systems, the natural solution of Eq. 1 is

$v=\frac{R}{2}\left(-f+\sqrt{f^{2}+\frac{4 \operatorname{grad} p}{R \rho}}\right)$

and the natural solution of Eq. 2 is

$v=\frac{R}{2}\left(f-\sqrt{f^{2}-\frac{4 \operatorname{grad} p}{R \rho}}\right)$.

The solutions given in Eq. 3 and Eq. 4 describe the gradient wind approximation in which the air blows parallel to the isobars leaving the high pressures on the right (BuyBallot law). Note that here we only consider the simplest situation where the square root argument is positive and the analytical solutions are real. Moreover, limit values of the centrifugal force that lead to other wind solutions such as the geostrophic or the cyclostrophic approximations (Willoughby, 1990) are not addressed here. A more 
complete treatment of the gradient wind equation solutions can be found in Knox and Ohmann (2006).

In this computational modelling activity we propose to students the computation and visualization of the gradient wind velocity. The first action is to select the Objects ribbon and create an Image object. This opens a new Animate ribbon panel (Fig. 5) where the user is asked to choose an image file and to adjust the scales of the horizontal and vertical coordinates to fit the Image in the workspace area. In this example the image chosen as background represents an idealized mean sea-level pressure distribution chart containing two schematic low pressure and high pressure systems (Fig. 6). The image frame, in latitude and longitude degrees, provides the scale.

The first exercise consists of placing an air particle object on the low pressure system and to build an animation of the particle's trajectory as a function of time. The initial position of the particle determines the radius of curvature $R$ (distance from the low pressure centre) and the local magnitude of the pressure gradient (pressure difference between two adjacent isobars divided by the distance between them), which must be estimated beforehand based on the pressure distribution chart.

The trajectory of an air particle can be described using polar coordinates $r=R$ and $\theta$ $=\omega \mathrm{t}$, where $\omega=v / R$ is the gradient wind angular velocity. Students must first write in the Mathematical Model window the expressions for the velocity and coordinates $X$ and $y$ (Fig. 6). The next step is to introduce in the Parameters ribbon the estimated values of the air density $\rho$, the Coriolis parameter $f$, the value of the local pressure gradient magnitude gradp and the radius of curvature $R$ of the trajectory.

Students can now place a Particle object at a distance $R$ from the centre of the low pressure system. In the Particle Animate panel (Fig. 5) it suffices to assign $x$ as the Particle horizontal coordinate and $y$ as its vertical coordinate, and to adjust the scale. Note that in order to obtain a good simulation an appropriate time step must be defined in the Independent Variable ribbon, in this case $\Delta t=1000 \mathrm{~s}$ (Fig. 6). Running the model shows the air particle describing a counter clockwise circular motion around the low pressure centre leaving higher pressures on the right. Finally, the value of the gradient wind speed can be visualized in the workspace using a Variable object $(\mathrm{V})$ placed at the bottom of the figure.

The second stage of the activity involves the computation and visualization of the air particle velocity and of the acceleration vectors corresponding to the Coriolis force $\left(\vec{a}_{\text {Cor }}\right)$, the pressure gradient force $\left(\vec{a}_{\mathrm{p}}\right)$ and the centrifugal force $\left(\vec{a}_{\mathrm{c}}\right)$. Students must then add to the mathematical model the $\mathrm{x}$ and $\mathrm{y}$ components of these vectors (for example $a_{\mathrm{px}}$ and $a_{\mathrm{py}}$ ), which in the low pressure system are respectively given by

$\vec{v}=v \overrightarrow{u_{\theta}}, \quad \vec{a}_{\text {Cor }}=f v \overrightarrow{u_{r}}, \quad \vec{a}_{p}=-\frac{\operatorname{grad} p}{\rho} \overrightarrow{u_{r}}, \quad \vec{a}_{c}=\frac{v^{2}}{R} \overrightarrow{u_{r}}$,

where $\overrightarrow{u_{r}}=(x, y) / R$ and $\overrightarrow{u_{\theta}}=(-y, x) / R$. Students can now create Vector objects to represent the velocity and the accelerations (Fig. 6). This is done in the Vector Animate ribbon (Fig. 5) assigning the $x$ components to the Vectors horizontal coordinates and the $y$ components to their vertical coordinates, and adjusting each one of the Vector scales for appropriate visualization. Next, students should attach the Vectors to the Particle (Figs. 5 and 6). Re-running the model, students can verify that the gradient wind velocity is always tangent to the circular trajectory and the accelerations are perpendicular to the gradient wind velocity, the pressure gradient acceleration pointing inward towards the low pressure centre and the Coriolis and the centrifugal accelerations pointing outward to the high pressures. To check the acceleration range of 
magnitudes students can define these magnitudes in the Mathematical Model window and visualize them in the Animation using Variable objects (Fig. 6).

Following the same steps students can easily construct a similar mathematical model and animation for the clockwise circular motion of an air particle around the high pressure system. An example of the class support documentation given to students to complete this exercise is given in the supplementary material. The support documentation is given in the form of an editable PDF file. It contains two parts: the first part consists of step-by-step instructions on how to implement the low pressure gradient wind model; the second part is an example of an incomplete activity where students are asked to change the mathematical equations and display the high pressure gradient wind animation. The solution of the incomplete activity is shown in Figure 6 where we can see an air particle and the corresponding acceleration vectors moving around the high pressure. The analysis of the force-balance in both the low and high pressure systems is very instructive for students, since they verify themselves that the pressure gradient force and the Coriolis force change directions but the centrifugal force is always directed outward the curve. They can also check interactively that the wind velocity in the high pressure system is smaller due to the smaller pressure gradient force, that is, due to the larger isobar spacing.

\section{Discussion and Conclusions}

Over the years several computer modelling systems, for example, the Dynamical Modelling System (Ogborn and Wong, 1984), Stella (Richmond, 2004), Coach (Heck et al., 2009), EJS (Christian and Esquembre, 2007) and Modellus (Teodoro and Neves, 2011), have been developed to introduce scientific computation without requiring the development of a working knowledge of programming. This is a problem which inevitably arises with professional programming languages such as Fortran (Chapman, 2007), Pascal (Redish and Wilson, 1993), Java (Gould et al., 2007) or Python (Chabay and Sherwood, 2008), professional scientific computation software such as Mathematica or Matlab, or even with educational programming languages like Logo (Papert, 1980) or Boxer (diSessa, 2000).

Among such computer modelling systems, Modellus has been proving particularly useful to teach introductory physics and mathematics (see, e.g., Neves et al., 2010, 2011; Neves and Teodoro, 2010; Teodoro and Neves, 2011) because it allows explorative to expressive modelling (Bliss and Ogborn, 1989; Schwartz, 2007) involving the simultaneous manipulation and analysis of several different model representations, namely, tables, graphs and animations with interactive objects whose properties are defined in a visible and modifiable mathematical physics model. Using Modellus modelling environment, students can thus create and explore models and animations with a computer, and not just act as simple browsers of computer simulations.

In this paper we have discussed how Modellus can be used to develop computational modelling activities within an interactive engagement teaching approach which introduce mathematical physics models of interest in geosciences contexts to students with only basic secondary level knowledge of physics and mathematics and no prior knowledge of scientific computation. As illustrative examples, with insights on Modellus functionalities and potentialities for computer-assisted teaching and learning, we have described activities about the blackbody radiation laws and the gradient wind dynamics. These, and other computational modelling activities, have been field tested in introductory meteorology courses we have implemented for first cycle undergraduate university students. As evidenced by the content analysis of student coursework and 
evaluation tests, the computational modelling activities contributed successfully to identify and resolve many of the student difficulties in key physics, scientific computation and meteorology concepts and processes. For example, the easy-to-draw Planck radiation curves helped students to better associate temperature with radiation emission and the gradient wind circular motion animation with wind particle acceleration vectors helped them make a vivid image of wind circulation. And this was done by the students themselves which constituted an extra motivation for learning.

To evaluate student's opinions about Modellus and the interactive computational modelling activities we used as instrument the Likert scale questionnaire shown in Fig. 7. The questionnaires were administered by us in class to all enrolled students at the end of the courses. Since 2009, in each academic year the introductory meteorology courses gathered an average of 50 students, all of which answered the questionnaire. As showed by the average results of Likert scale questionnaires (Fig. 7), the majority of students reacted very positively to the computational modelling activities with Modellus. The graph bars in Fig. 7 show the distribution over the Likert scale of the average student opinion per questionnaire assertion. The data was taken from the 2011 edition of the course which involved 53 students. These results show that students considered the activities useful for the learning processes in meteorology and for their professional training as a whole. In addition, Modellus was considered helpful and user friendly. Finally, though less strongly, students reacted favourably to work in collaborative groups and using Modellus in other geosciences subjects with appropriate computational modelling activities.

\section{Acknowledgments}

Work partially supported by UIED, FCT/UNL and Fundação para a Ciência e a Tecnologia, Ministério da Educação e Ciência (FCT/MEC), Programa Compromisso com a Ciência, Ciência 2007. We also thank the valuable comments of three anonymous reviewers who helped to improve the manuscript.

\section{References}

Beichner, R., Saul, J., Abbott, D., Morse, J., Deardorff, D., Allain, R., Bonham, S., Dancy, M., Risley, J., 2007. The student-centered activities for large enrollment undergraduate programs (SCALE-UP) project. In: Redish, E., Cooney, P. (Eds.), Research-Based Reform of University Physics, Reviews in Physics Education Research Vol. 1, American Association of Physics Teachers, College Park, MD, USA, 42pp. http://www.per-central.org/document/ServeFile.cfm?ID=4517 [accessed in July 27, 2012].

Bliss, J., Ogborn, J., 1989. Tools for exploratory learning. Journal of Computer Assisted Learning 5, 37-50.

Blum, W., Galbraith, P., Henn, H.-W., Niss, M. (Eds.), 2007. Modelling and Applications in Mathematics Education: The $14^{\text {th }}$ ICMI Study, Springer, New York, USA, 521pp.

Chabay, R., Sherwood, B., 2008. Computational physics in the introductory calculusbased course. American Journal of Physics 76, 307-313. 
Chapman, S., 2007. Fortran 95/2003 for Scientists and Engineers (3rd ed.). McGrawHill. 1008pp.

Christian, W., Esquembre, F., 2007. Modeling physics with Easy Java Simulations. The Physics Teacher 45, 475-480.

Crouch, C., Watkins, J., Fagen, A., Mazur, E., 2007. Peer instruction: Engaging students one-on-one, all at once. In: Redish, E., Cooney, P. (Eds.), Research-Based Reform of University physics, Reviews in Physics Education Research Vol. 1, American Association of Physics Teachers, College Park, MD, USA, 55pp. http://www.percentral.org/document/ServeFile.cfm?ID=4990 [accessed in July 27, 2012].

diSessa, A., 2000. Changing Minds: Computers, Learning and Literacy. MIT Press, Cambridge, MA, USA, 293pp.

Gould, H., Tobochnik, J., Christian, W., 2007. An Introduction to Computer Simulation Methods: Applications to Physical Systems. Addison-Wesley, San Francisco, CA, USA, 796pp.

Halloun, I., Hestenes, D., 1985. The initial knowledge state of college physics students. American Journal of Physics 53, 1043-1056.

Halloun, I., Hestenes, D., 1985. Common-sense concepts about motion. American Journal of Physics 53, 1056-1065.

Handelsman, J., Ebert-May, D., Beichner, R., Bruns, P., Chang, A., DeHaan, R., Jim Gentile, J., Lauffer, S., Stewart, J., Tilghman, S., Wood, W., 2005. Education: Scientific teaching. Science 304, 521-522.

Heck, A., Kadzierska, E., Ellermeijer, T., 2009. Design and implementation of an integrated computer working environment. Journal of Computers in Mathematics and Science Teaching 28, 147-161.

Klosko, E., DeLaughter, J., Stein, S., 2000. Technology in introductory geophysics: the high-low mix. Computers and Geosciences 26, 693-698.

Knox, J., Ohmann, P., 2006. Iterative solutions of the gradient wind equation. Computers and Geosciences 32, 656-662.

Kortz, K., Smay, J., Murray, D., 2008. Increasing student learning in introductory geoscience courses using Lecture Tutorials. Journal of Geoscience Education 56, 280290.

Libarkin, J., Anderson, S., 2005. Assessment of Learning in Entry-Level Geoscience Courses: Results from the Geoscience Concept Inventory. Journal of Geoscience Education 53, 394-401.

Libarkin, J., Anderson, S., Dahl, J., Beilfuss, M., Boone, W., Kurdziel, J., 2005. Qualitative analysis of college students' ideas about the Earth: Interviews and openended questionnaires. Journal of Geoscience Education 53, 17-26. 
Mazur, E., 1997. Peer Instruction: A User's Manual. Prentice Hall, Upper Saddle River, NJ, USA, 253pp.

McConnell, D., Steer, D., Owens, K., Borowski, W., Dick, J., Foos, A., Knott, J., Malone, M., McGrew, H., Van Horn, S., Greer, L., Heaney, P., 2006. Using Concept Tests to assess and improve student conceptual understanding in introductory geoscience courses. Journal of Geoscience Education 54, 61-68.

McDermott, L., 1991. Millikan lecture 1990: What we teach and what is learned closing the gap. American Journal of Physics 59, 301-315.

McDermott, L., Shaffer, P., Rosenquist, M., 1996. Physics by Inquiry: An Introduction to Physics and the Physical Sciences. Wiley, New York, USA, 864pp.

McDermott, L., Shaffer, P., 2002. Tutorials in Introductory Physics, Prentice Hall, New Jersey, USA, 244pp.

McDermott, L., Redish, E., 1999. Resource letter PER-1: Physics education research. American Journal of Physics 67, 755-767.

Meltzer, D., Thornton, R., 2012. Resource Letter ALIP-1: Active-learning instruction in physics. American Journal of Physics 80, 478--496.

Mintzes, J., Wandersee, J., Novak, J. (Eds.), 2005. Teaching Science for Understanding: A Human Constructivist View. Elsevier Academic Press, Burlington, MA, USA, 360pp.

Neves, R., Silva, J., Teodoro, V., 2010. Computational modelling in science, technology, engineering and mathematics education. In: Araújo, A., Fernandes, A., Azevedo, A., Rodrigues, J. (Eds.), Proceedings of the EIMI 2010 Conference:

Educational Interfaces between Mathematics and Industry, Centro Internacional de Matemática and Comap Inc., Bedford, MA, USA, pp. 387-397.

Neves, R., Silva, J., Teodoro, V., 2011. Improving learning in science and mathematics with exploratory and interactive computational modelling. In: Kaiser, G., Blum, W., Borromeo-Ferri, R., Stillman, G. (Eds.), International Perspectives on the Teaching and Learning of Mathematical Modelling Vol. 1, ICTMA14 - Trends in Teaching and Learning of Mathematical Modelling, Springer, Dordrecht, pp. 331-341.

Neves, R., Teodoro, V., 2010. Enhancing science and mathematics education with computational modelling. Journal of Mathematical Modelling and Application 1, 2-15.

Ogborn, J., Wong, D., 1984. A microcomputer dynamical modelling system. Physics Education 19, 138-142.

Papert, S., 1980. Mindstorms: Children, Computers and Powerful Ideas. Basic Books, New York, USA, 230pp.

Redish, E., Saul, J., Steinberg, R., 1998. Student expectations in introductory physics. American Journal of Physics 66, 212-224 
Redish, E., Wilson, J., 1993. Student programming in the introductory physics course: MUPPET. American Journal of Physics 61, 222-232.

Richmond, B., 2004. An Introduction to Systems Thinking with Stella. ISEE Systems Inc., Lebanon, NH, USA, 165pp.

Schwartz, J., 2007. Models, simulations, and exploratory environments: A tentative taxonomy. In: Lesh, R., Hamilton, E., Kaput, J. (Eds.), Foundations for the Future in Mathematics Education, Lawrence Erlbaum Associates, Mahwah, NJ, USA, pp. 161172.

Teodoro, V., Neves, R., 2011. Mathematical modelling in science and mathematics education. Computer Physics Communications 182, 8-10.

Willoughby, H., 1990. Gradient balance in tropical cyclones. Journal of the Atmospheric Sciences 47, 265-274.

\section{Figures with Captions}

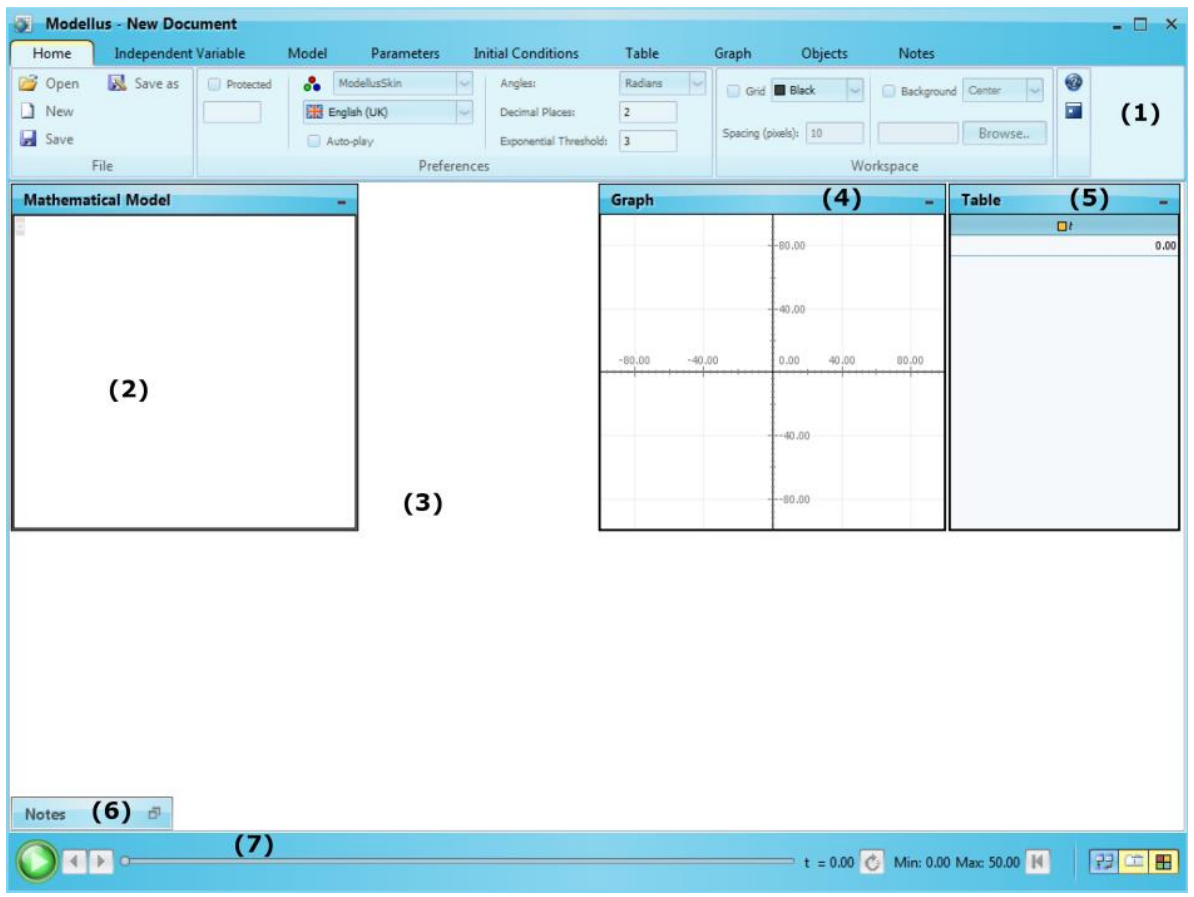

Figure 1: Modellus window interface showing (1) Home ribbon which can be changed selecting a different ribbon label, (2) Mathematical Model window, (3) Animation area, (4) Graph window, (5) Table window, (6) Notes window and (7) Animation Control bar. 


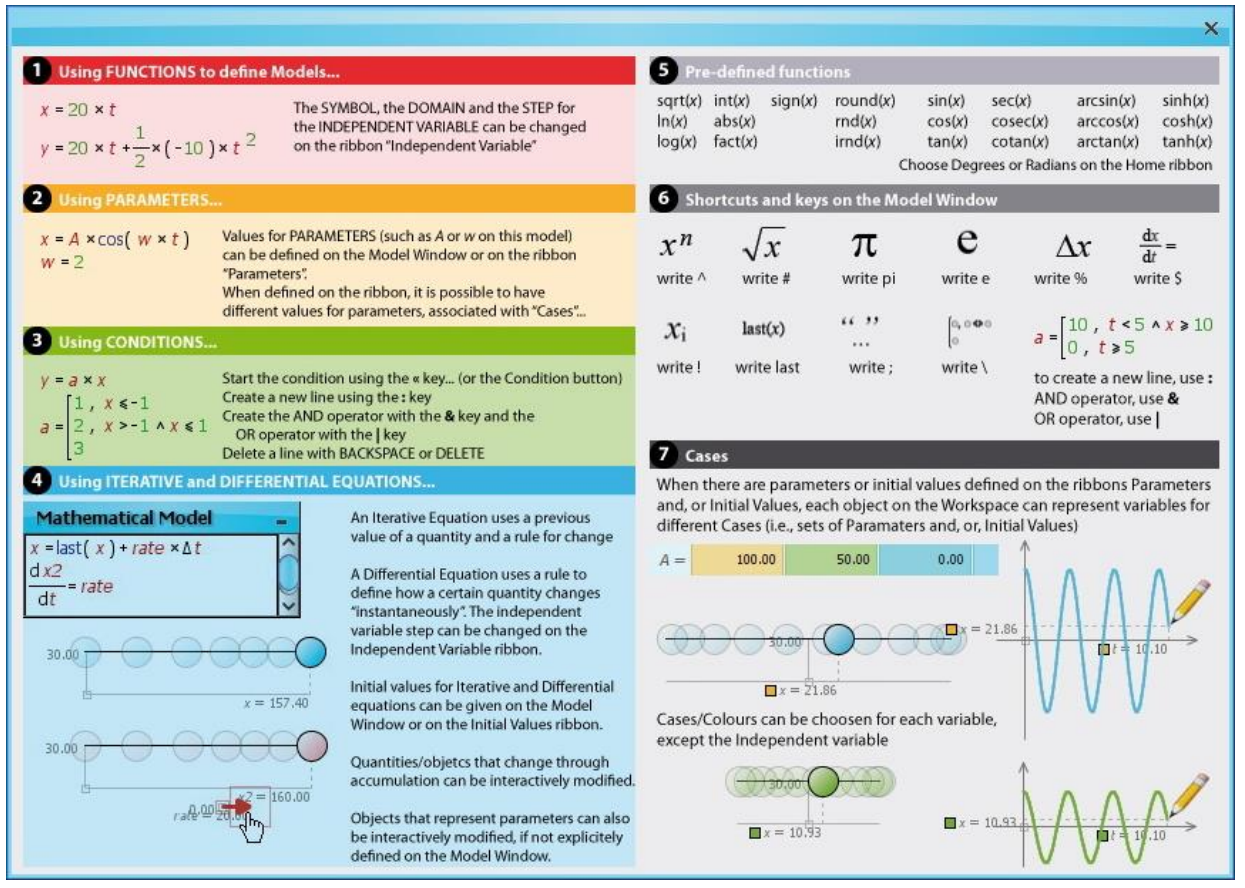

Figure 2: Modellus Help page with instructions for building 4 different mathematical models, lists of pre-defined function and keyboard shortcuts, and an example involving different cases.

\begin{tabular}{|c|c|c|c|c|c|c|c|c|c|c|c|}
\hline Home & Independ & it Variable & Model & Para & & Initial Conditions & & Table & Graph & Objects & Notes \\
\hline$c=$ & $3.00 E 8$ & 3.00EB & $3.00 E B$ & 0,00 & 0.00 & 0.00 & 0.00 & 0.00 & 0.00 & 0.00 & \begin{tabular}{|l|l} 
All equal & A
\end{tabular} \\
\hline$k=$ & $1.38 \mathrm{E}-23$ & $1,38 E-23$ & $1,38 \mathrm{E}-23$ & 0.00 & 0.00 & 0.00 & 0.00 & 0.00 & 0,00 & 0.00 & $\square$ All equal $\square$ \\
\hline$T=$ & 500.00 & 400,00 & 300.00 & 0.00 & 0.00 & 0.00 & 0.00 & 0.00 & 0.00 & 0.00 & $\square$ All equal $\checkmark$ \\
\hline \multicolumn{12}{|c|}{ Parameters } \\
\hline
\end{tabular}
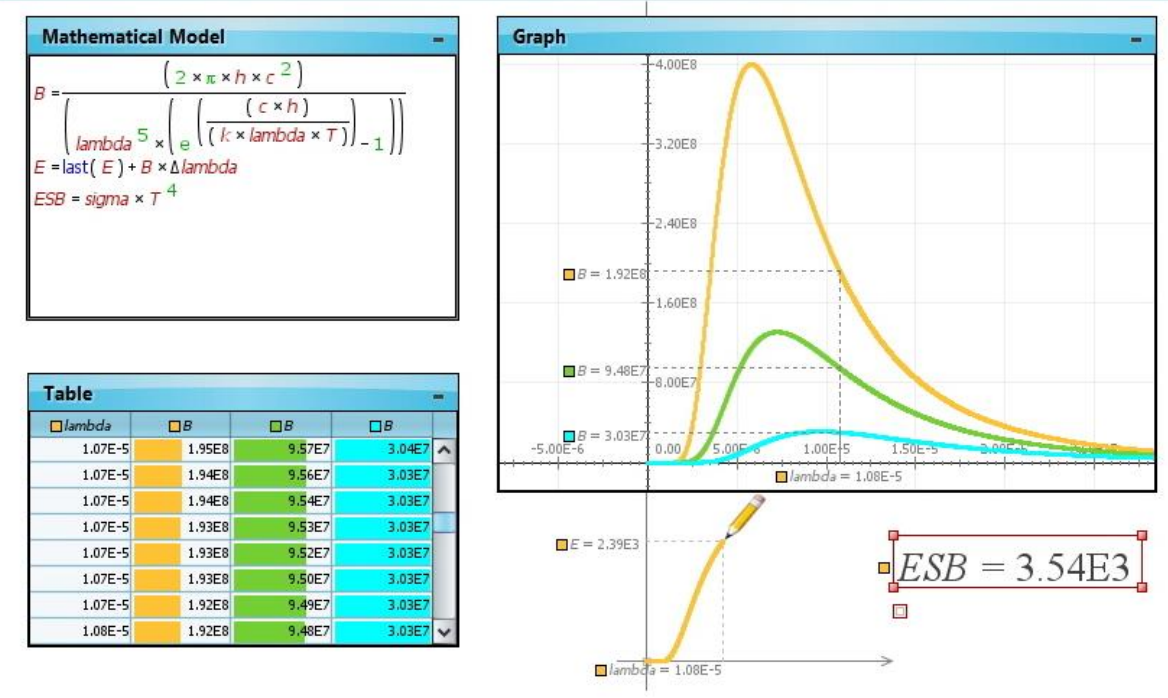

Figure 3: Modellus blackbody radiation model showing Mathematical Model window with Planck's radiation power density function $B(\lambda)$ and its numerical integration over wavelength $\lambda$, denoted as lambda, leading to Stefan-Boltzmann law for $E$, power radiated per unit area. Also shown are Graph window with 3 curves for 3 different temperatures, $T=500 \mathrm{~K}$ (orange), $T=400 \mathrm{~K}$ (green) and $T=300 \mathrm{~K}$ (cyan), Table window and Animation with Pen showing graph of $E$ as function of $\lambda$ and Variable ESB displaying Stefan-Boltzmann limit $\sigma T^{4}$ for $T=500 \mathrm{~K}$. 

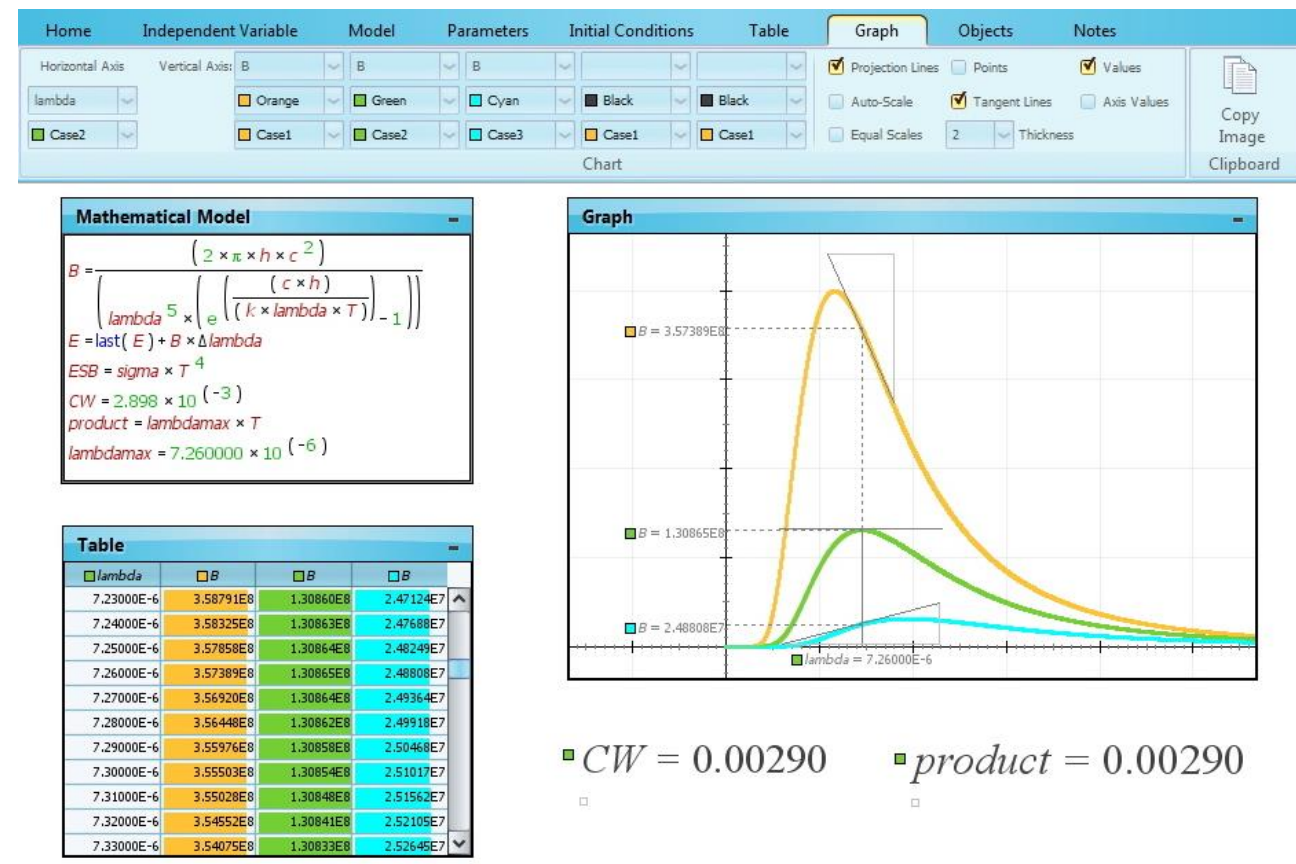

${ }^{\square} C W=0.00290 \quad$ a product $=0.00290$

Figure 4: Modellus graphical deduction of Wien displacement law using a tangent line to $T=400 \mathrm{~K}$ Planck density curve. To move tangent line along the curve select and hold down left mouse button or drag Independent Variable button in Animation Control bar. To select maximum in curve increase number displaying precision selecting at least 5 decimal places in Home ribbon. Then use One step back or One step forward buttons and check values in Table window.
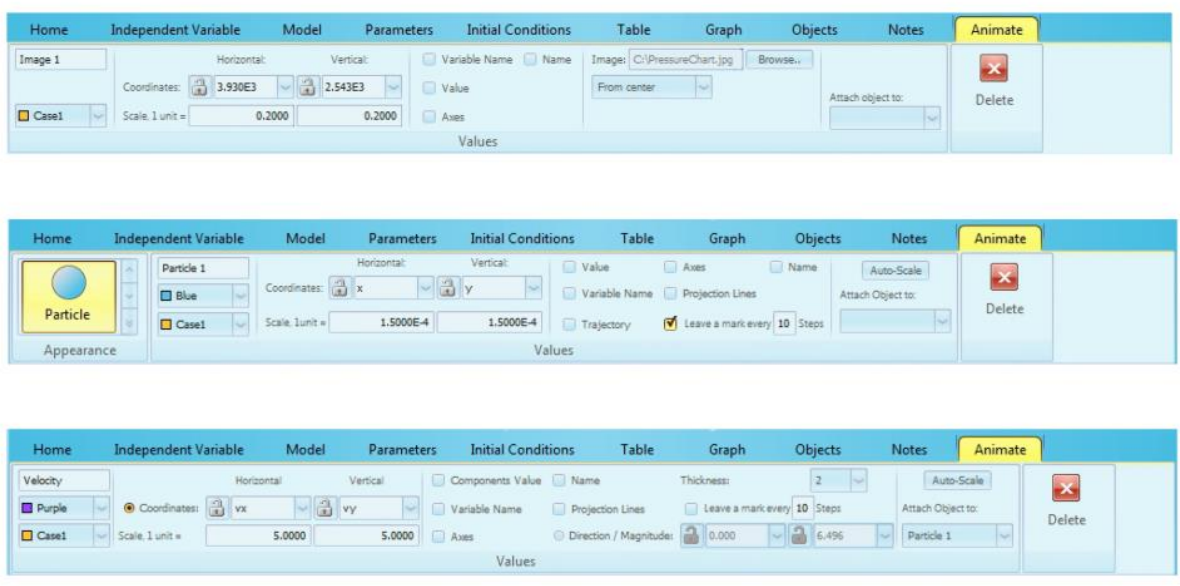

Figure 5: Modellus Animate panels for Image, Particle and Velocity Vector Animation Objects. 


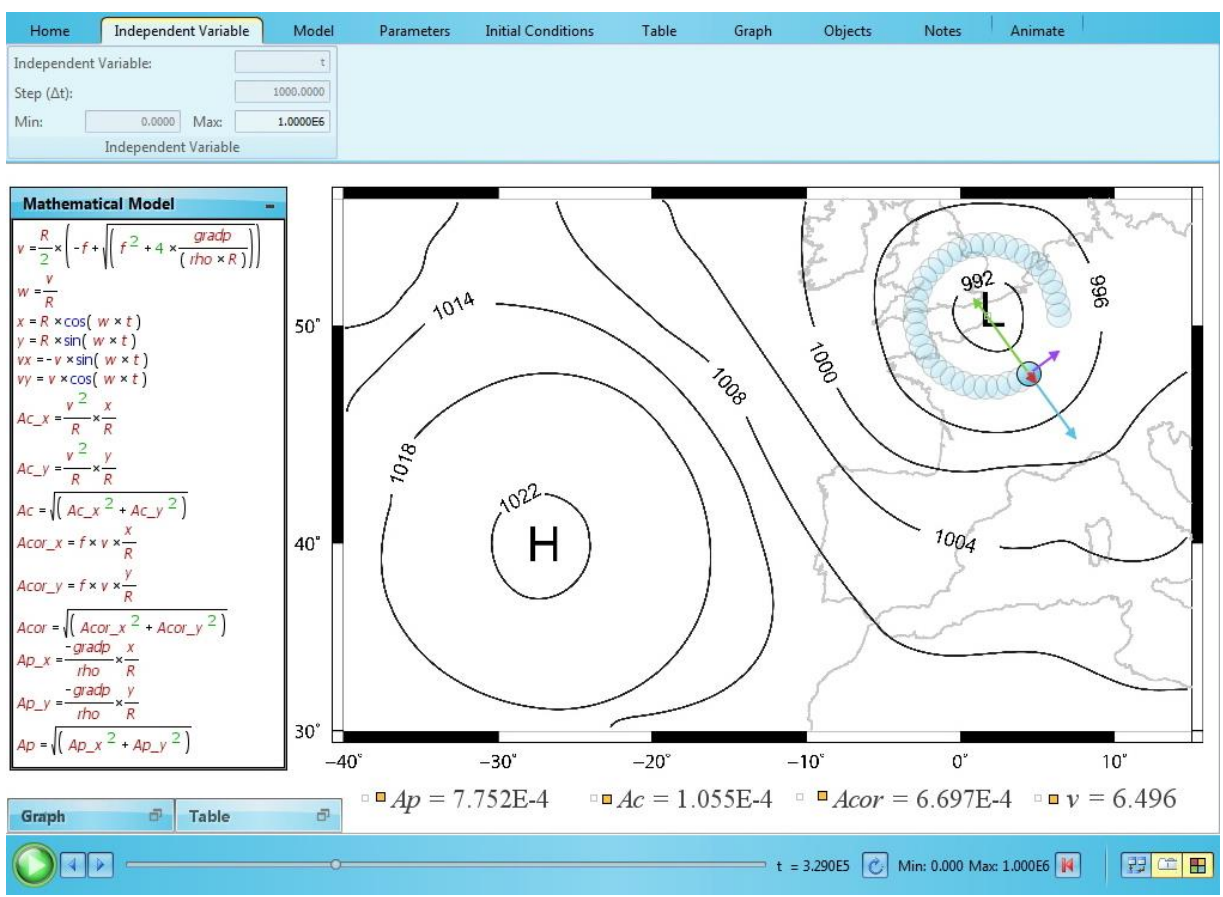

Figure 6: Modellus gradient wind velocity model showing Mathematical Model window with low pressure natural velocity solution, air particle position coordinates and Coriolis, pressure gradient and centrifugal accelerations. An idealized mean sea-level pressure distribution chart Image is used as background for air Particle counter clockwise circular motion around low pressure centre. Attached to Particle are gradient wind velocity (purple), Coriolis acceleration (blue), pressure gradient acceleration (green) and centrifugal acceleration (red) Vectors. Their magnitudes are shown below the Image chart in Variable objects. Model parameters are $R=400 \mathrm{~km}, \rho=1.29 \mathrm{~m}^{-3}$, $\operatorname{grad} p=0.001 \mathrm{~Pa}$ and, for latitude $\varphi=50$ degrees North, $f=1.031 \mathrm{E}-4 \mathrm{~s}^{-1}$. An air particle revolving around the high pressure is also shown as the solution of an incomplete activity given to students. The all activity is included in the PDF supplementary file given as an example of the class support documentation.

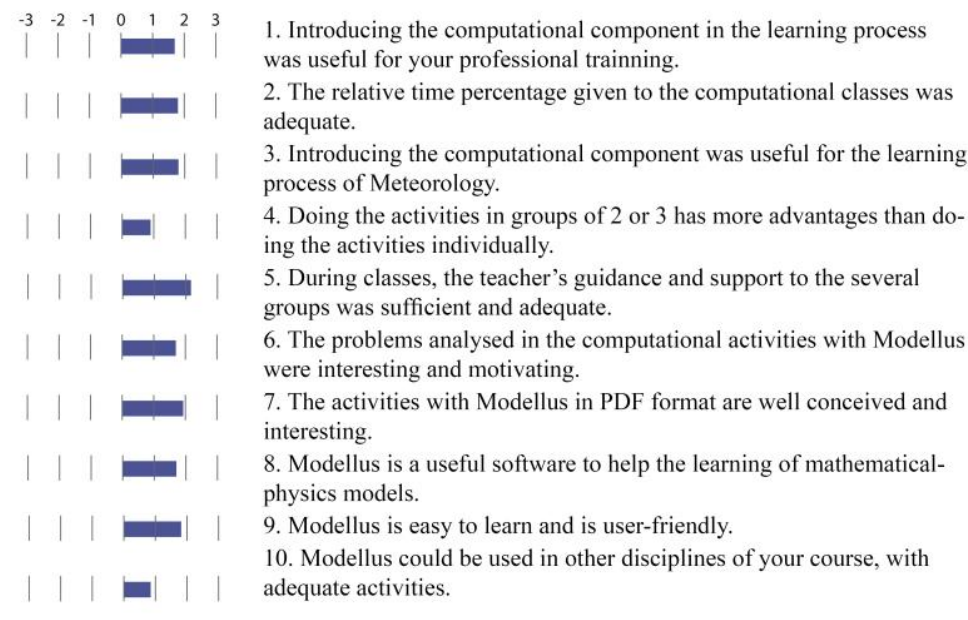

Figure 7: Introductory meteorology student opinion questionnaire and results. For each questionnaire assertion Likert scale starts at -3 and ends at $+3,-3$ stating complete disagreement, +3 complete agreement and 0 no preferred opinion. Bar graph shows distribution over Likert scale of average student opinion per questionnaire assertion. 